Int. J. Curr. Res. Med. Sci. (2016). 2(12): 27-32

\begin{tabular}{|c|c|c|}
\hline 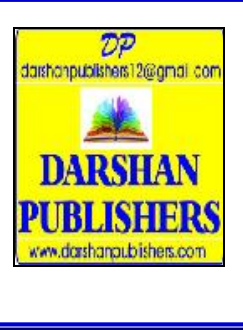 & $\begin{array}{l}\text { International Journal of Current Research in } \\
\text { Medical Sciences } \\
\text { ISSN: 2454-5716 } \\
\text { Www.ijcrims.com } \\
\text { Volume 2, Issue 12 -2016 }\end{array}$ & 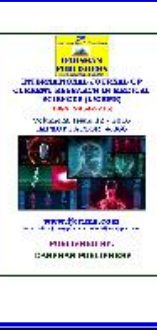 \\
\hline
\end{tabular}

Case study

DOI: http://dx.doi.org/10.22192/ijcrms.2016.02.12.005

\title{
Extensor pollicis longus superficialis and extensor indicis superficialis, can they be considered as a new anatomical variation in the long extensors of fingers?
}

\author{
Ahmed Farid Al-Neklawy, M.D. \\ Anatomy and Embryology Department, Faculty of Medicine, Ain Shams University, Cairo, Egypt \\ E-mail: dr_ahmed_farid@yahoo.com, Tel: 00201001850336
}

Running title: Two extra muscles for thumb and index fingers

\begin{abstract}
Background: Variations of anomalies of hand extensors have been described by many authors. These anomalies are often discovered during routine surgical procedures and cadaveric dissections. Being aware of such anomalies is important to the physician in order to avoid unintentional damage to healthy tendons during surgical procedures. In addition, accessory tendons have the potential to be used in the surgical repair or replacement of damaged tendons. We reported a cadaveric case with bilateral two additional superficial extensors to the thumb and index fingers with unique features. The names of extensor pollicis longus superficialis (EPL-S) and extensor indicis superficialis(EI-S) were proposed.

Methods: A female cadaver was used in this study. Bilateral dissection of the forearm and wrist was done.

Results: Two extra muscles were observed in the superficial group of the extensors of the forearm. They were situated between extensor carpi radialis brevis and extensor digitorum muscles. Both muscles originated from the common extensor origin. One muscle was inserted into the distal phalanx of the thumb, while the other was inserted into the extensor expansion of the index finger.

Conclusion: These findings improve that variations of the extensor muscles and tendons of the hand are not uncommon. Anatomists and physicians should be aware of these anomalies, as they may cause physical symptoms and signs such as swelling and pain related to the hand.
\end{abstract}

Key words: Extensor musculature anomalies, extensor pollicis longus variations, extensor indicis variations, variations of long extensor tendons.

\section{Introduction:}

Many variations of anomalies of hand extensor muscles have been described by several authors over the last century and half, starting with Wood [11].
Awareness of the existence of such anatomical variations is clinically useful in the field of the planning of tendon transfers or repairs. More while, there have been many clinical reports of physical signs and symptoms such as swelling and 
pain resulting from the existence of supernumerary extensor tendons, which can often be misdiagnosed due to unawareness of such variations $[2,6,9]$.

Variations of the extensor tendons to thumb and index have been reported and become valuable in cases requiring transfer or repair of these tendons. These variations are often discovered during routine surgical procedures or cadaveric dissections. Currently available reports on these variations mostly focus on the short extensor tendons of thumb and index. So far, only a few papers have been published describing variations of the long extensor tendons of the thumb and index [1-4].

\section{Methods:}

In this study, we present a cadaveric case in which there were two independent superficial extensor muscles for thumb and index fingers arising from the common extensor origin and passing through the third and fourth dorsal synovial compartments respectively. These variations have been found bilaterally during routine dissection of a female cadaver for use in a graduate gross anatomy laboratory course. For precise descriptive purposes, the names extensor pollicis longus superficialis (EPL-S) and extensor indicis superficialis (EI-S) were proposed. The specimen was formaldehyde preserved, and this is why the quality of the dissection is not good.

\section{Case representation:}

During routine dissection of the right forearm and wrist of the female cadaver, aberrant two extra muscles were observed originating from the common extensor origin, and were situaded between the extensor carpi radialis brevis (ECRB) and extensor digitorum(ED) muscles (Fig. 1).

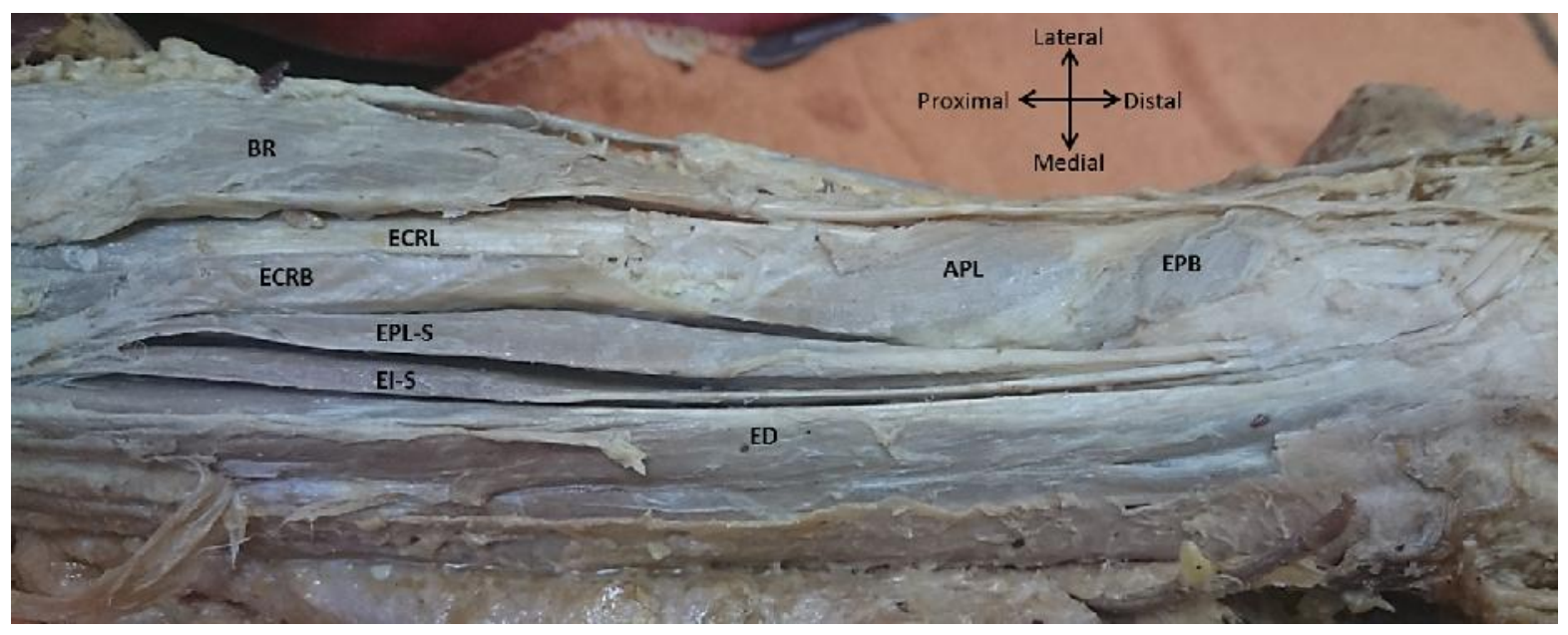

Fig.1: A photograph of dorsal view of the right forearm showing the two extra muscles EPL-S and EI-S situated between the ECRB and ED muscles, and originating from the common extensor origin of the forearm. BR brachioradialis, ECRL extensor carpi radialis longus, ECRB extensor carpi radialis brevis, EPL-S extensor pollicis longus superficialis, EI-S extensor indicis superficialis, ED extensor digitorum, APL abductor pollicis longus, EPB extensor pollicis brevis.

Following the tendons of the muscles distally, it was found that the tendon of the lateral extra muscle (EPL-S) passed through the dorsal third synovial compartment and had a parallel course medial to the extensor pollicis longus (EPL) till the level of the metacarpophalangeal joint of the thumb, where it crossed from medial to lateral superficial to the EPL tendon to be inserted independently into the dorsal aspect of the base of the terminal phalanx of the thumb (Figs. 2 and 3). 


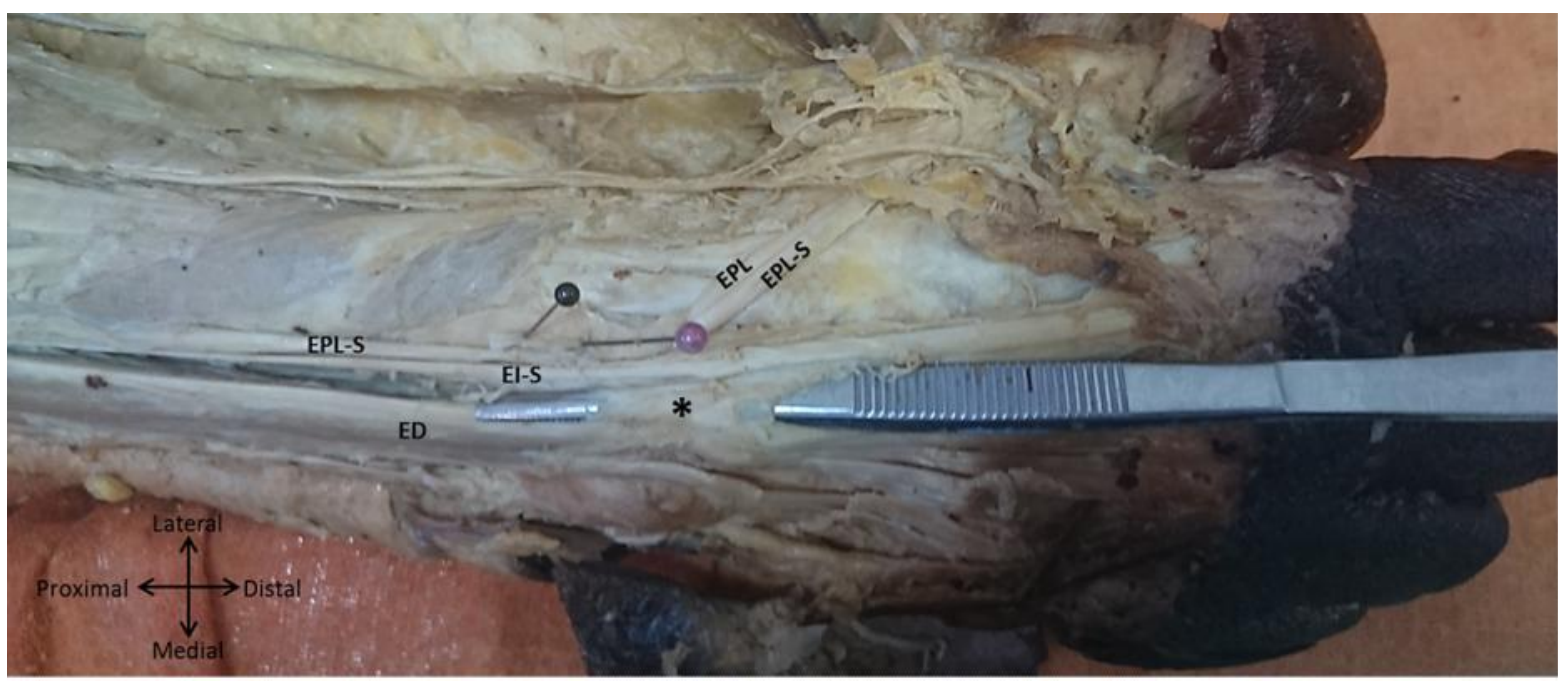

Fig. 2: A photograph of dorsal view of the right wrist showing the tendons of two extra muscles EPL-S and EI-S. the tendon of EPL-S is passing through the dorsal third synovial compartment and deviates laterally parallel to the tendon of EPL. The tendon of EI-S is passing through the dorsal fourth synovial compartment, and separated from the other tendons of ED which are enclosed in a separate fascial sheath (*). EPL-S extensor pollicis longus superficialis, EI-S extensor indicis superficialis, ED extensor digitorum, EPL extensor pollicis longus.

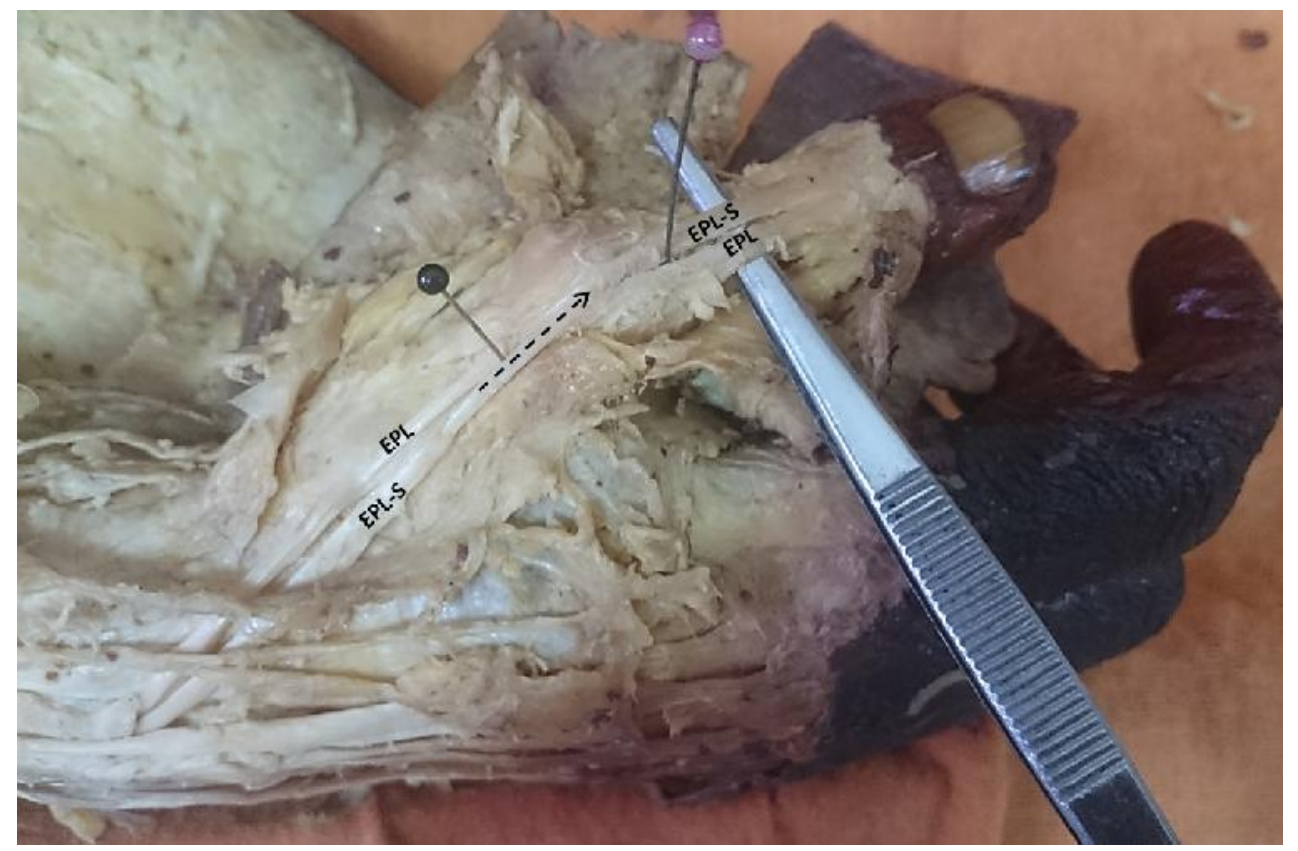

Fig. 3: A photograph of dorsolateral view of the right wrist showing the tendon of EPL-S passing medial and parallel to the tendon of EPL down to the level of the metacarpophalangeal joint of the thumb, then it passes superficial to the tendon of EPL from medial to lateral (dotted arrow), to be inserted separately into the base of the distal phalanx of the thumb. EPL-S extensor pollicis longus superficialis, EPL extensor pollicis longus.

While the tendon of the medial extra muscle (EIS) passed through the dorsal fourth synovial compartment within a special fascial sheath separating it from the ED muscle (Fig. 2), and had a course lateral and parallel to the tendon of extensor indicis (EI) muscle to be inserted into the extensor expansion of the index finger. The tendon of EI muscle was splitted into two parts 
(Figs. 4, and 5).The tendon of ED of the index finger was absent, giving a hypothesis of the extra muscle of the index that it was early separated from extensor digitorum muscle, but being enclosed in a special fascial sheath has promoted the idea of existence of a separate muscle to the index finger.

Dissection of the left forearm and wrist in this cadaver revealed the presence of the two extra muscles with the same findings mentioned before.

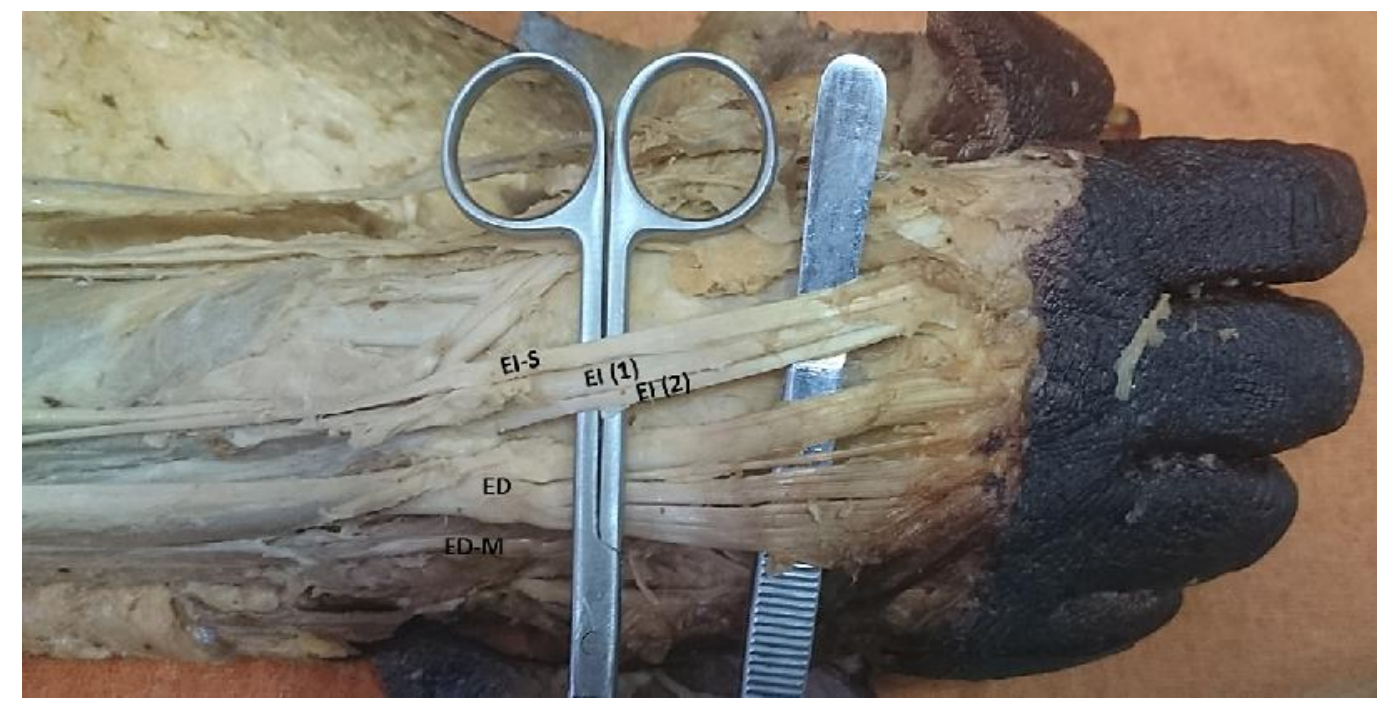

Fig. 4: A photograph of dorsal view of the right wrist showing the tendon of EI-S passing lateral and parallel to the tendon of EI which is splitted into two parts EI(1) and EI(2). Notice the absence of the extensor diditorum tendon of the index finger. EI-S extensor indicis superficialis, EI(1) extensor indicis lateral slip, EI(2) extensor indicis medial slip, ED extensor digitorum, ED-M extensor digiti minimi.

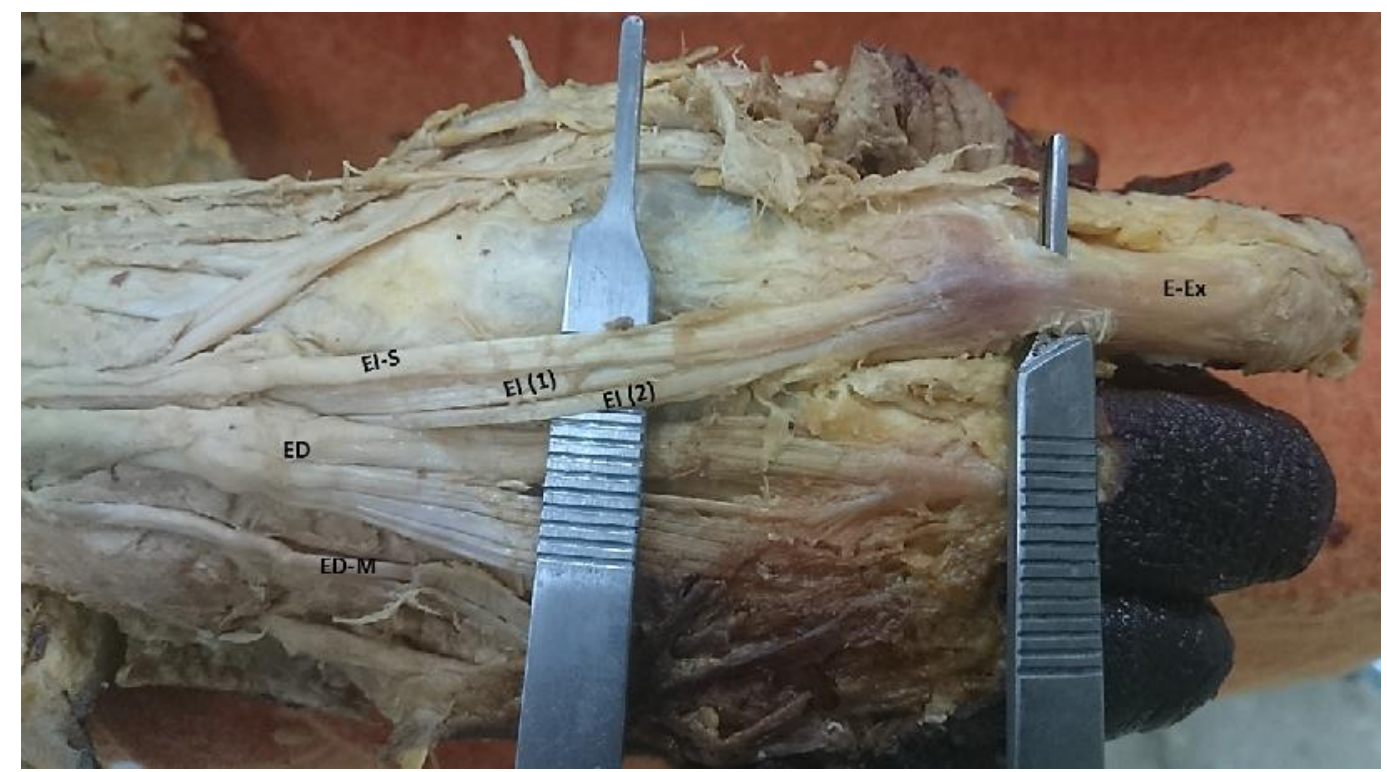

Fig. 5: A photograph of dorsal view of the right wrist showing the tendon of EI-S passing lateral and parallel, then superficial to the tendon of EI which is splitted into two parts EI (1) and EI(2). Notice the common insertion of these tendons into the extensor expansion of the index finger. EI-S extensor indicis superficialis, EI(1) extensor indicis lateral slip, EI(2) extensor indicis medial slip, ED extensor digitorum, ED-M extensor digiti minimi, E-Ex extensor expansion. 


\section{Discussion}

Anomalies of hand and forearm musculature are common and varied. In the case of the EPL, there have been several reports of accessory muscle bellies and supernumerary tendons, as well as the presence of tendons branching from the EI or ED that parallel the course of the EPL tendon [4].

Abu-Hijileh [1] has reported the first case of an extra extensor muscle and tendon to the right thumb. The muscle was discovered during routine dissection of a male cadaver. It originated from the dorsal aspect of the lower third of ulna, just proximal to the origin of extensor indicis muscle. Its tendon was inserted independently into the base of the proximal phalanx of the thumb after passing through the dorsal fourth synovial compartment.

Tolga et al [10] have described a classification system for anomalies of the EPL. They have reported three cases in which there was a supernumerary long extensor tendon to the thumb. Their classification was built according to details of the anomalies are described along with a metaanalysis of relevant literature. They also concluded that in all cases, the supernumerary tendon emerged from the fourth dorsal compartment and had a parallel course to the EPL.

Cazanova et al [3] have described an extensor indicis radialis muscle with fascial connection with the tendon of extensor pollicis longus tendon.

However, to our knowledge, this is the first report that describes such two complete independent muscles for both thumb and index fingers, originated superficially from the common extensor origin. In addition to the course of the tendon of EPL-S which crossed from medial to lateral superficial to the tendon of EPL tendon after its passage through the third dorsal synovial compartment, to be inserted into the base of the distal phalanx of the thumb.
The development of these anomalies is likely due to alteration in either the formation of specific muscle masses by myoblasts migrating into the distal limb or by alteration in the muscle patterning dictated by the somatopleure mesoderm [5].

This variation, as with other extensor anomalies, may cause physical symptoms and signs such as swelling and pain with hand dysfunction, such as a third and fourth compartments syndrome, and may require significant consideration in hand surgery. There may be a role for magnetic resonance imaging scanning of patients suspected to have these variations to help in diagnosis, or to be used as a preoperative screen before surgical exploration $[\mathbf{2}, \mathbf{7}, \mathbf{8}]$.

\section{Conflict of interest:}

The author declares that he has no conflict of interest, commercial associations, or intent of financial gain regarding this research.

\section{References}

1. Abu-Hijleh MF. (1993): Extensor pollicistertius: an additional extensor muscle to the thumb. Plast Reconstr Surg. 92(2):340343.

2. Beatty JD, Remedios D, McCullough CJ. (2000): An Accessory Extensor Tendon of the Thumb as a Cause of Dorsal wrist Pain. J Hand Surg. 25B: 110-11.

3. Casanova Martínez D, Valdivia Gandur I, Golanó P. (2013): Extensor pollicis et indicis communis or extensor indicis radialis muscle. Anat Sci Int. Jun; 88(3):153-5.

4. Chiu DT. (1981): Supernumerary extensor tendon to the thumb: a report on a rare anatomic variation. Plast Reconstr Surg. 68(6):937-939.

5. Christ B, Brand-Saberi B. (2002): Limb muscle development. Int J Dev Biol. 46:905914.

6. Gahhos FN, Ariyan S. (1983): Extensor indicis brevis: a rare anatomic variation. Ann Plast Surg. 10: 326-8. 
7. Masada K, Yasuda M, Takeuchi E, et al. (2003): Duplicate extensor tendons of the thumb mimicking rupture of the extensor pollicis longus tendon. Scand J Plast Reconstr Surg Hand Surg. 37(5):318-19.

8. Sookur PA, Am N, Bleakney RR, et al. (2008): Accessory muscles: anatomy, symptoms, and radiologic evaluation. Radio Graphics. 28:481-490.

9. Tan ST, Smith PJ. (1999): Anomalous extensor muscles of the hand: a review. J Hand Surg [Am]. 24: 449-55.

10. TolgaTürker, Gregory A. Robertson, and Sunil M. Thirkannad (2010): a classification system for anomalies of the extensor pollicis longus. Hand (N Y). 5(4): 403-407.

11. Wood J. (1864): On some varieties in human myology. Proc R Soc Lond.13:299-303.

\begin{tabular}{|c|l|}
\hline \multicolumn{2}{|c|}{ Access this Article in Online } \\
\hline & Website: \\
\hline & www.ijcrims.com \\
\hline Quick Response Code & \\
\hline
\end{tabular}

How to cite this article:

Ahmed Farid Al-Neklawy. (2016). Extensor pollicis longus superficialis and extensor indicis superficialis, can they be considered as a new anatomical variation in the long extensors of fingers?. Int. J. Curr. Res. Med. Sci. 2(12): 27-32.

DOI: http://dx.doi.org/10.22192/ijcrms.2016.02.12.005 